\title{
A๒1100 ๆ8ьนเ?
}

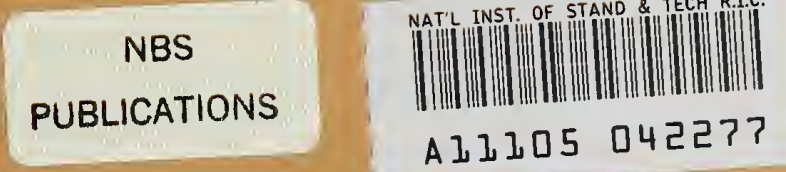

\section{CONTAINER \\ BOARD}

report no. 118

July 1979

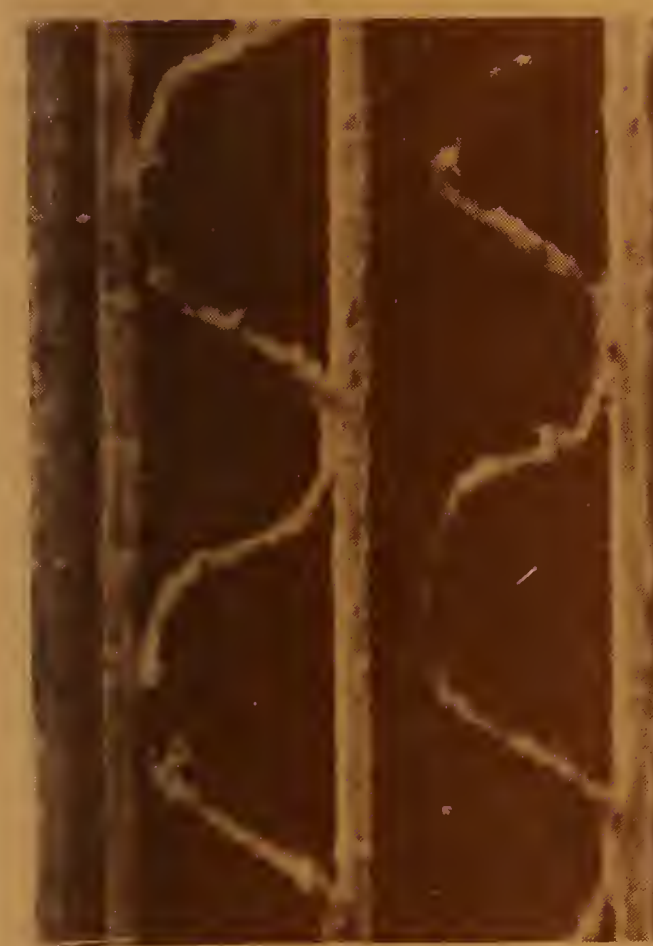

NBS Collaborative Reference Program for Containerboard

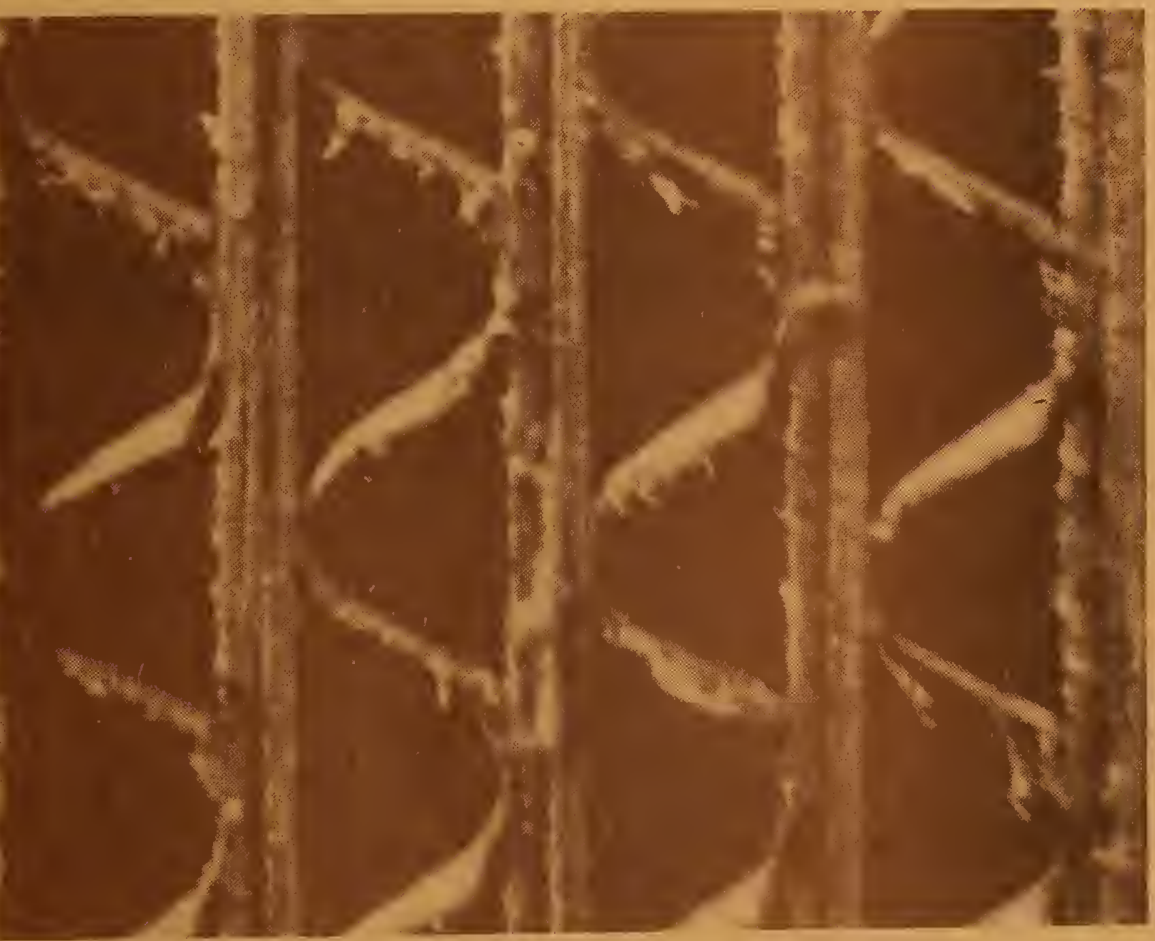

Fourdrinier Kraft Board Group American Paper Institute, Inc. and U.S. Department of Commerce, National Bureau of Standards

$Q C$

100

.456

$79-1809$

1979

C.2 


\section{TAPPI Paper and Board ( 6 times per year)}

Bursting strength

Tearing strength

Tensile breaking strength

Elongation to break

Tensile energy absorption

Folding endurance

Stiffness

Air resistance

Grammage
Smoothness

Surface pick strength

$\mathrm{K} \& \mathrm{~N}$ ink absorption

$\mathrm{pH}$

Opacity

Blue reflectance (brightness)

Specular gloss, $75^{\circ}$

Thickness

Concora (flat crush)

Ring crush

FKBG-API Containerboard (48 times per year)

Mullen burst of linerboard

Concora test of medium

MCCA Color and Appearance (4 times per year)

Gloss at $60^{\circ}$

Color and color difference

CTS Rubber (4 times per year)

Tensile strength, ultimate elongation and tensile stress Hardness

Mooney viscosity

Vulcanization properties

CTS Thermal Insulation Materials (2 times per year)

19 test methods for thermal insulation materials covering: thermal properties; strength properties; dimensions, stability, and density properties; fire properties; and properties of vapor barriers

ASTM Cement (2 times per year)

Chemical' (11 chemical components)

Physical ( 8 characteristics)

\section{AASHTO Bituminous}

Asphalt cement (2 times per year)

Cutbacks (once a year)

NBS Collaborative Reference Programs A05 Technology Building National Bureau of Standards Washington, DC 20234 


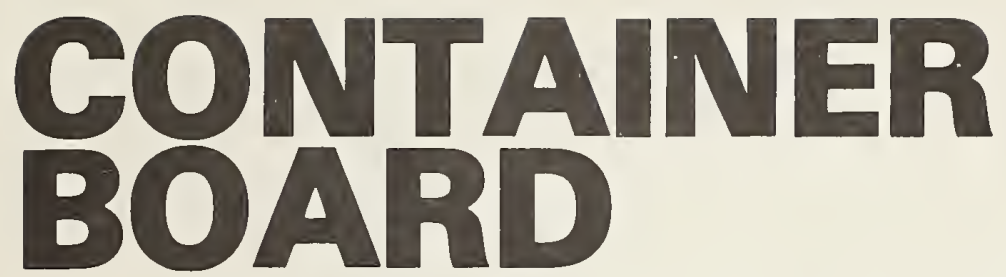

\section{Collaborative Reference report no. 118 Program for Containerboard \\ July 1979}

\section{T.L. Cummings}

NBS Research Associate Collaborative Testing Services, Inc.

J. Horlick

Office of Testing Laboratory

Evaluation Technology,

Office of Engineering Standards, National Engineering Laboratory

U.S. Department of Commerce, Fourdrinier Kraft Board Group National Bureau of Standards American Paper Institute, Inc. 



\section{INTRODUCTION}

The Collaborative Reference Program for Containerboard is cosponsored by the Fourdrinier Kraft Board Group (FKBG) of the American Paper Institute, Inc. and the National Bureau of Standards. The program is maintained and operated by Collaborative Testing Services, Inc. (CTS). CTS is a non-profit organization of associations that offers CRPs to a wide range of industries.

Samples of three weights of linerboard, nominally $26 \mathrm{lb}, 42 \mathrm{lb}$, and $69 \mathrm{lb}$ and of corrugating medium (26 lb) are separately randomized from uniform narrow rolls and packaged for distribution to the participants. Each month, sufficient test material for four weekly tests is mailed to participants for testing Mullen bursting strength according to TAPPI official testing method T807 os-75 or Concora flat crush strength according to TAPPI official testing method T809 os-71. The participants return their test results to NBS for analysis and receive two monthly reports from NBS. One report is a preliminary individualized report comparing a laboratory's results with the industrial mean. The other is a longer report (as illustrated by this report) showing the data from all participants.

If there are any questions on the notes, the analyses, or the reports in general, contact Thomas L. Cummings or Jeffrey Horlick on (301) 921-2946.
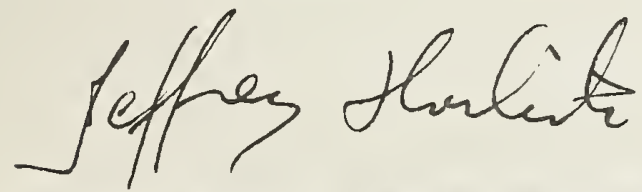

Jeffrey Horlick, Technical Administrator

NBS Collaborative Reference Programs

Office of Testing Laboratory Evaluation Technology

September 17, 1979 



\section{TABLE OF CONTENTS}

$\underline{\text { Page }}$

1 Explanation of Tables

4 Instrument Codes

5 Use of Average Mean as a Reference Standard

6 Bursting Strength, Linerboard 42H12, weeks 1 - 4

8 Bursting Strength, Linerboard 69J2, weeks $1-4$

10 Concora Flat Crush, Corrugating Mediun 26D2 

Each table shows laboratory test results for Mullen bursting strength of linerboard or Concora flat crush strength of corrugating medium. The data are divided into three time spans. On the left of each table is an analysis for each week of the month. In the center is cumulative data for the month and on the right is cumulative data for up to 16 weeks.

Conservative statistical tests have been used in excluding extreme data from the analyses. Thus, where the mean (average) for one laboratory is compared with the average for many laboratories, limits have been used that would exclude only one laboratory in a hundred if all laboratories followed exactly the same testing procedure. Consequently, laboratories receiving " $X$ " flags should review their testing procedures, instrument calibration, and control processes. Similar conservative criteria were used in flagging within-laboratory standard deviations and other statistics.

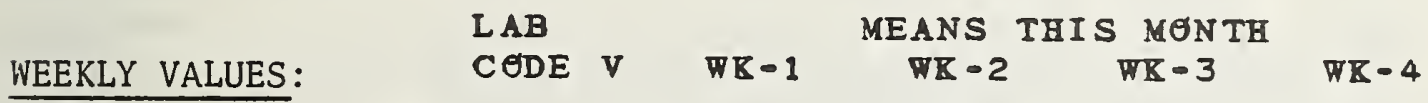

LAB CODE - Confidential laboratory identification number known only to the participant and the Collaborative Reference Program staff.

V - Code for indicating instrument type, units used, and any other variation in test procedure or conditions. A ' + ' in this column means a non-standard variation. Data marked ' + ' are not included in the combined averages for all laboratories. (see page 4).

MEANS THIS MONTH - For each laboratory each weekly mean is the average of individual test determinations, usually an average of 20 determinations.

FLAGS (following means and standard deviations) -

$X$ - Data excluded from an AV MEAN or average standard deviation because value deviated from the AV MEAN or average standard deviation by more than 2.576 times the appropriate standard deviation. A laboratory following the prescribed test method could obtain such an extreme value by chance only one time in a hundred. Corrective action is almost certainly required.

Data included in the CUMULATIVE AV MEAN but the value deviated from this mean by more than 1.960 and less that 2.576 times the SD CUM MEAN. A laboratory following the prescribed test method could obtain such an extreme value by chance only one time in twenty. Corrective action may be desired. 
$S$ - This is a warning to the laboratory but does not affect inclusion or exclusion of the laboratory's results from the corresponding AV MEAN. This flag indicates an extremely high or low within-laboratory standard deviation (SDR, not shown) that could occur by chance only one time in a hundred if the laboratory is following the prescribed test method.

AV MEAN - (at bottom of table) - The average for the indicated week of the means for all laboratories, except those laboratories marked ' +' in column $\mathrm{V}$ and those means marked with an ' $\mathrm{X}$ '.

SDR - (not shown) - The standard deviation of within-laboratory measurements; i.e., the Standard Deviation of the Replicate measurements made at one time in one laboratory on one package of test pieces.

AV SDR - The average for the indicated week of the SDR's of all the laboratories, except those omitted from the AV MEAN. Also an extremely high or low SDR as compared with the AV SDR based on the remaining laboratories is omitted from the AV SDR and the letter ' $S$ ' is placed after the laboratory mean for that week. The AV SDR is an index of the within-laboratory precision for repeated measurements; i.e., a measure of the ability of an average laboratory to repeat its results over a short period of time. It includes measurement error and sample variation.

SD LABS - For each week the standard deviation of the means about the AV MEAN for that week after omitting those means marked with an ' $X$ ' or noted $1+1$ in column $V$. The SD LABS is an index of the among-laboratory precision of the test method as applied by the participating laboratories; i.e., a measure of the ability of laboratories to get comparable results.

NO. INCL - The number of laboratory means included in the AV MEAN for that week.

NO. OMIT - The number of laboratory means reported but omitted from AV MEAN because of non-standard equipment, environment or procedure $('+1$ in column V) or because of extreme results ( $X$ following mean).

NOT RCD - The number of laboratories failing to report data on time or in usable form for this week (but who reported data for at least one of the other weeks of this month), or who received test pieces from a different sample of material and whose data therefore are shown in another table of this report.

SD SHTS - (Concora only) The average for the indicated week of the amongsheet within-laboratory standard deviations. The SD SHTS is an index primarily of the variability among sheets. 
VALUES THIS MONTH:

MEAN SDR SDWRS

MEAN - The average for the indicated laboratory of the reported weekly MEANS THIS MONTH.

SDR - The average for the indicated laboratory of the weekly SDRs for the current month.

SDWKS - For the indicated laboratory, the standard deviation among the laboratory's weekly MEANS THIS MONTH (including those means marked with an ' $X '$ ').

\section{CUMULATIVE VALUES:
MEAN SDR SIVE}

MEAN - The average for the indicated laboratory of all its weekly means for the number of weeks indicated, including those for the current month. An ' $*$ ' or ' $X$ ' following this CUMULATIVE MEAN indicates the laboratory is running consistently low or high. (See above for explanation of these flags).

SDR - The average for the indicated laboratory of the weekly SDRs for the indicated number of weeks.

SDWKS - For the indicated laboratory, the standard deviation among the laboratory's weekly means (including those means marked with an ' $X$ '). SDWKS is an index of the week to week precision; i.e., a measure of the ability of a laboratory to repeat its results from week to week.

WKS - Number of weeks for which usable results have been reported by that laboratory. At most, 16 weeks of data are included.

\section{GRAND AVERAGES \\ GRAND AVERAGES: THIS MENTH CUMULATIVE 12 WEEKS}

THIS MONTH - Averages for the four weeks of the quantities shown to the left.

CUMULATIVE - Averages for the indicated number of weeks, including the four weeks of the current month. 
AV SDWKS - The average of the SDWKS for all laboratories excluding those marked ' + ' in column $V$ or with an ' $X$ ' following the corresponding THIS MONTH or CUMULATIVE MEAN or SDWKS.

SD CUM MEAN - The larger of either (1) the standard deviation of the CUMULATIVE MEANS about the average CUMULATIVE MEAN after omitting those CURSULATIVE MEANS marked with an ' $X$ ' or with $a^{i}+1$ in column $V$, or (2) the CUMULATIVE SD LABS divided by the square root of the number of weeks cunulated. The former will be appreciably larger than the latter only when there are persistent systematic differences among the laboratories.

\section{INSTRUMENT CODES}

FOR

MULLEN BURST TESTERS

(Column V)

Code

Description

A

Model A, Manual Clamp

H

Model AH, Hydraulic Clamp

I

Model A, Hydraulic Clamp added

J

Jumbo, Hand Clamp, Hand Driven

L

Lhomargy, Hydraulic Clamp

M

Model AH, Hydraulic Clamp, Transducer

R

Model A, Air Clamp added

$x$

Other Model, Please Describe Instrument Make and Model

If an incorrect instrument code has been assigned to your laboratory, please inform us. 
A large supply of linerboard in three weights was randomized and placed in sealed packages ready for shipment. The supply for each weight of board was divided into several narrow "rolls" or cross-machine "positions" of a larger roll, and each position was separately randomized. Each package contains test pieces from one position only. The position is designated by the number following the letter in the code marked on the package. Thus $42 \mathrm{H} 1$ indicates that this package contains $421 \mathrm{~b}$ board from position 1 of lot $H$. Samples from the first position are distributed until exhausted, then from the second position, and so forth for each weight of board. Thus for short periods of time (several weeks to months), the samples that the participants test are from the same position of a lot, and for a longer period from the same lot.

The three weights of linerboard distributed in this program may be used as reference standards. The best reference values are the cumulative grand AV MEANs in the latest reports. These values are given at the bottom right of each table. For each weight of board, comparisons should be made first for measurements made on the same position, i.e., for checking your current measurement, use grand AV MEANs that have the same position code as on the packages being tested. The position is shown in the upper left corner of the table. If no report is yet available on the current position, grand AV MEANs from previously tested positions of the same lot may be used as approximate reference values.

Similarly a large supply of a 26 lb corrugating medium was randomized, after dividing into several narrow rolls or positions. The above discussion for linerboard also applies to the corrugating medium.

We are currently using the third lot of linerboard and the fourth lot of corrugating medium:

\begin{tabular}{|c|c|c|c|}
\hline Lot & Material & Codes & Used \\
\hline 1 & linerboard & $A, B, C$ & October 1969 - Apri1 1973 \\
\hline 2 & linerboard & $D, E, F$ & September 1972 - September 1976 \\
\hline 3 & linerboard & $\mathrm{G}, \mathrm{H}, \mathrm{I}, \mathrm{J}$ & October 1976 - \\
\hline 1 & corrugating medium & (A) & May 1973 - March 1976 \\
\hline 2 & corrugating medium & $\mathrm{B}$ & April 1976 - February 1977 \\
\hline 3 & corrugating medium & $\mathrm{C}$ & March 1977 - August 1978 \\
\hline 4 & corrugating medium & $\mathrm{D}$ & September 1978 - \\
\hline
\end{tabular}


BURSTING STRENGTH (MULIEN), PSI

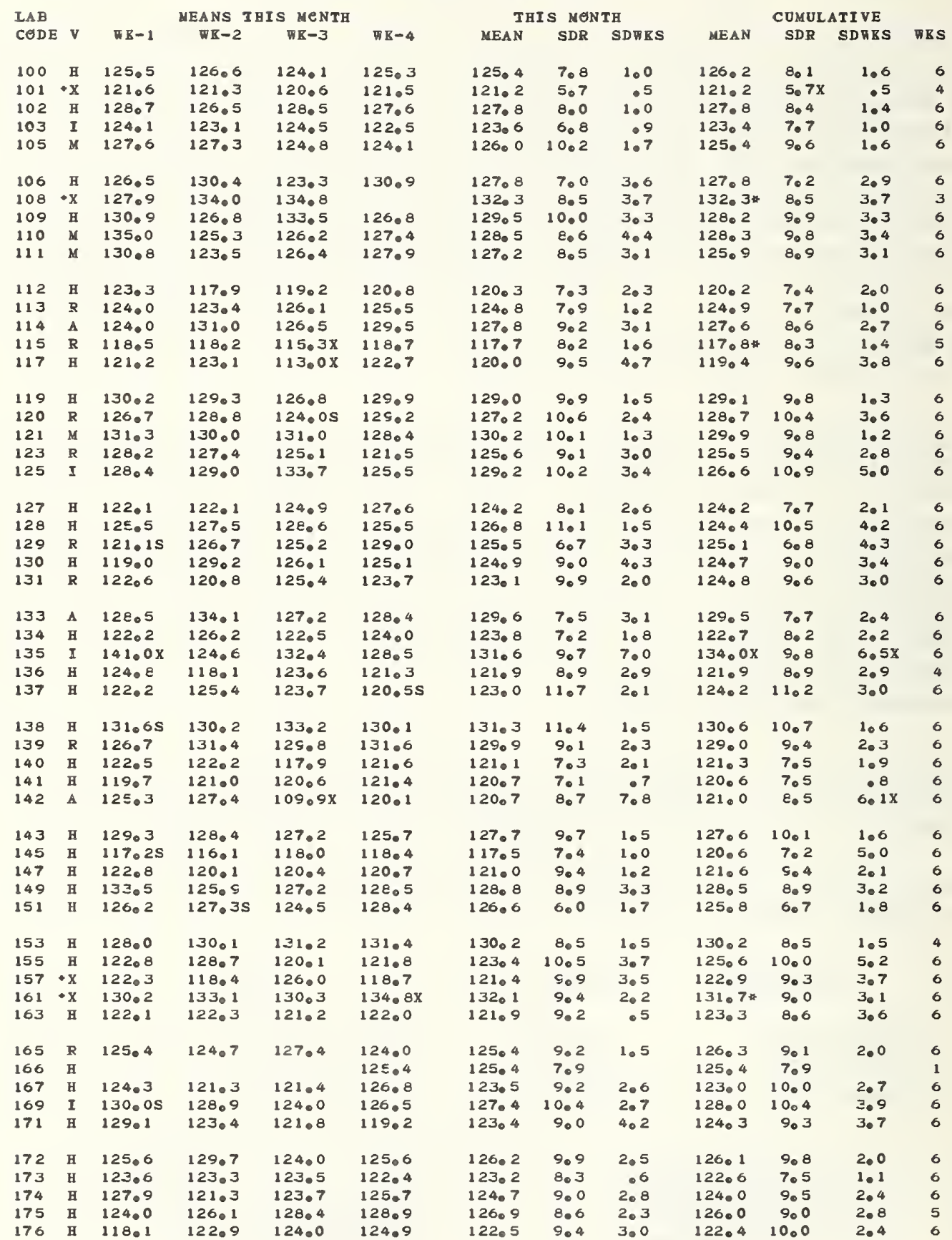


BURSTING STRENG TH (MULLEN), PSI

\begin{tabular}{|c|c|c|c|c|c|c|c|c|c|c|c|c|}
\hline \multirow{2}{*}{$\begin{array}{l}L A B \\
C O D E\end{array}$} & \multirow{2}{*}{$E \mathbf{v}$} & \multicolumn{4}{|c|}{ MEANS IBIS MENTB } & \multicolumn{3}{|c|}{ IEIS MONTH } & \multicolumn{4}{|c|}{ CUYULATIVE } \\
\hline & & E-1 & W $\mathrm{B}$-2 & N $\mathrm{B}=3$ & $m x-4$ & MEAN & SDR & SDFRS & NEAN & SDR & SDWKS & WRS \\
\hline 177 & H & $119 \cdot 5$ & $119 \cdot 6$ & $122 \cdot 8$ & 120.7 & 120.7 & 8.0 & 1.5 & 119.7 & 7.7 & 2,1 & 6 \\
\hline 182 & H & $123 \cdot 4$ & 122.5 & $127 \cdot 8$ & 127.9 & $125 \cdot 4$ & 8.1 & 2.8 & $125 \cdot 5$ & e. 1 & 2.2 & 6 \\
\hline 184 & $\mathbf{H}$ & $126 \cdot 1$ & $124 \cdot 7$ & $125 \cdot 3$ & $131 \cdot 7$ & 127.0 & 7.7 & $3 \cdot 2$ & $128 \cdot 0$ & 8.0 & 3.1 & 6 \\
\hline 186 & $\mathbf{I}$ & $121 \cdot 7$ & $124 \cdot 2$ & 120.5 & $124 \cdot 5$ & 122.8 & 8.2 & 2.0 & $122 \cdot 3$ & 7.7 & 1.7 & 6 \\
\hline 188 & I & $122 \cdot 4$ & $126 \cdot 6$ & $126 \cdot 8$ & $126 \cdot 2$ & 125,5 & 7.7 & 2.1 & 125.1 & 708 & 1.9 & 6 \\
\hline 250 & - I & 13001 & $127 \cdot 8$ & $121 \cdot 0$ & 122.4 & $125 \cdot 3$ & 1001 & $4 \cdot 3$ & 125,3 & 10.1 & 4.3 & 4 \\
\hline 274 & H & 125.9 & $125 \cdot 7$ & $125 \cdot 2$ & $125 \cdot 9$ & $125 \cdot 7$ & 7.5 & $\cdot 3$ & $125 \cdot 9$ & 7.6 & $\cdot 3$ & 6 \\
\hline 283 & H & 126.2 & 121.7 & $122 \cdot 7$ & $123 \cdot 8$ & 123.6 & $7 \cdot 2$ & 1.9 & $125 \cdot 3$ & 606 & $4 \cdot 1$ & 6 \\
\hline 287 & $\boldsymbol{\Delta}$ & $142.5 x$ & $143.5 \mathrm{X}$ & $149 \cdot 3 x$ & 147.0XS & 145. $6 x$ & 10.5 & 3.1 & $143.0 \mathrm{X}$ & 10.0 & $8 \cdot 0 x$ & 6 \\
\hline 350 & H & 119.4 & $123 \cdot 4$ & $128 \cdot 4$ & $129 \cdot 2$ & $125 \cdot 1$ & 10.2 & $4 \cdot 6$ & $123 \cdot 2$ & 10.1 & 4.7 & 6 \\
\hline 553 & $\mathbf{M}$ & $129 \cdot 5$ & 133,2 & 132.1 & 132,5 & 131.8 & 7.6 & 1.6 & $131 \cdot 7 *$ & 7.9 & $1 \cdot 4$ & 6 \\
\hline 562 & $\mathbf{A}$ & 134.9 & 134.8 & $143 \cdot 3 x$ & $141 \cdot 2 x$ & $138.6 x$ & 11.6 & 4.4 & $137 \cdot 7 x$ & 11.4 & 4.3 & 6 \\
\hline 568 & I & $122 \cdot 7$ & $125 \cdot 4$ & 125.9 & 128.0 & 125,5 & 1001 & $2 \cdot 2$ & 126.4 & 9.9 & 2,8 & 6 \\
\hline 569 & $\mathbf{A}$ & 132.7 & 129.0 & $140.7 x$ & $134.7 x$ & $134 \cdot 3 x$ & 9.3 & 4.9 & 132.3 내 & 9.1 & 409 & 6 \\
\hline 658 & H & 119.4 & 127.0 & $127 \cdot 3$ & $124 \cdot 5$ & 124.6 & 8.2 & 306 & 12403 & 8.4 & 3.6 & 6 \\
\hline 682 & H & $129 \cdot 7$ & $148 \cdot 2 x$ & $147.0 x$ & $145 \cdot 5 x$ & $142,6 x$ & 7.9 & 8.7 & $142.6 x$ & 7.9 & e.7x & 4 \\
\hline 701 & H & 129.4 & 127.1 & 125,6 & & 127.4 & 11.1 & 1.9 & 127.9 & 10.3 & 1.6 & 5 \\
\hline
\end{tabular}

\begin{tabular}{|c|c|c|c|c|c|c|c|c|c|c|}
\hline & 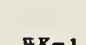 & $x=2$ & $x=3$ & & & THTS & GRAN L & AVERAGES & 6 & MEERS \\
\hline & $w 5-1$ & $x-2$ & $\mathrm{w} \mathrm{K}-3$ & $w s-4$ & & $1 \mathrm{H} 1 \mathrm{~s}$ & MONTH & & 0 & WEESS \\
\hline$\triangle V$ MEAN & $125 \cdot 5$ & 125,6 & 125.5 & 125.6 & $\mathbf{A V}$ & MEAN & $125 \cdot 6$ & 125.5 & & \\
\hline$\Delta V \quad$ SDR & 8.5 & 8.5 & $9 \cdot 1$ & 8.5 & $\Delta \mathbf{V}$ & SDR & 8.8 & 8.8 & & \\
\hline SD LABS & 4.2 & 4.0 & 3.7 & 3.5 & SD & IABS & 309 & 309 & & \\
\hline Ne. INCL & 64 & 64 & 59 & 62 & $N \theta_{0}$ & I NCL & $62 \cdot 2$ & 61.8 & & \\
\hline NO, OMI T & 7 & 7 & 12 & 8 & $\Delta \mathbf{V}$ & SDWRS & 2.7 & 2.6 & & \\
\hline NOT RCD & 1 & 1 & 1 & 2 & SD & CUM MEL & & 3. 1 & & \\
\hline
\end{tabular}



BURSING STRENGTH (MUILEN), PSI

\begin{tabular}{|c|c|c|c|c|c|c|c|c|c|c|c|c|}
\hline \multicolumn{2}{|l|}{ LAB } & \multicolumn{4}{|c|}{ MEANS IEIS MENTE } & \multicolumn{3}{|c|}{ IEIS MONTH } & \multicolumn{4}{|c|}{ COMULATIVB } \\
\hline$C \Theta D E$ & $\mathrm{v}$ & F $K-1$ & $\mathrm{~W} K=2$ & $w x-3$ & T $\mathrm{X}=4$ & MBAN & SDR & SDWES & MEAN & SDR & SDW KS & MR \\
\hline 100 & H & 154.1 & $155 \cdot 5$ & 158.0 & $157 \cdot 1$ & 15603 & 11.6 & 1.7 & $155 \cdot 2$ & $12 \cdot 5$ & 2.8 & 5 \\
\hline 101 & $+x$ & 152.6 & 153.9 & 157.9 & 156.7 & $155_{0} 3$ & 1309 & 2.4 & $154 \cdot 7$ & 13.0 & 2.5 & 5 \\
\hline 102 & H & 159.2 & $166 \cdot 5 x$ & $159 \cdot 2$ & 160.9 & $161 \cdot 4$ & $10 \cdot 2$ & 305 & 160.9 & 10.5 & $3 \cdot 2$ & 5 \\
\hline 103 & $\mathbf{I}$ & $15 e_{0} 8$ & $157 \cdot 4$ & $158 \cdot 3$ & 156.0 & $157 \cdot 6$ & 12.8 & $1 \cdot 2$ & 158.0 & 1301 & $1 \cdot 3$ & 5 \\
\hline 105 & $\mathbf{M}$ & 156.0 & $150 \cdot 1$ & 159.9 & $150 \circ 35$ & 154.1 & 17.5 & $4 \cdot 8$ & $154 \cdot 3$ & 16.7 & 4. 1 & 5 \\
\hline 106 & B & 149.0 & $151 \cdot 6$ & $155 \cdot 2$ & 156.7 & 153.1 & 9.7 & 3.5 & $154 \cdot 4$ & 9.5 & 4.1 & 5 \\
\hline 108 & $4 x$ & 161.4 & 164.2 & 154.0 & & 159.9 & $12 \cdot 7$ & $5 \cdot 3$ & 16004 & 12.5 & 4.4 & 4 \\
\hline 109 & H & 157.7 & 160.4 & 158.7 & 158.0 & 158.7 & 17.7 & $1 \cdot 2$ & $158 \cdot 4$ & 18.4 & 1.3 & 5 \\
\hline 110 & $\mathbf{M}$ & $155 \cdot 4$ & 152.0 & $154 \cdot 8$ & 160.6 & 155.7 & 15.0 & $3 \cdot 6$ & 155.8 & 15.8 & 3. 1 & 5 \\
\hline 111 & M & 155.9 & 152.9 & 152.9 & 154.4 & 15400 & 16.7 & 1.4 & 154.0 & 1603 & 1.2 & 5 \\
\hline 112 & H & $154 \cdot 3$ & 154.0 & $149 \cdot 2$ & $153 \cdot 1$ & 152.7 & 10.0 & $2 \cdot 4$ & $154 \cdot 2$ & 10.3 & $3 \cdot 9$ & 2 \\
\hline 113 & $\mathbf{R}$ & 156.7 & $156 \cdot 1$ & 157.9 & 156.3 & 156.7 & 10.7 & 08 & 156.6 & 10.7 & -8 & 5 \\
\hline 114 & $\mathbf{A}$ & $154 \cdot 2$ & $157 \cdot 5$ & $159 \cdot 2$ & 156.8 & 156.9 & 13.8 & $2 \cdot 1$ & $156 \cdot 8$ & 1304 & $1 \cdot 8$ & 5 \\
\hline 115 & $\mathbf{R}$ & 161.5 & 153.1 & $148 \cdot 2$ & 153.85 & $154 \cdot 2$ & 10.4 & $5 \cdot 5$ & $153 \cdot 8$ & 10.2 & $4 \cdot 8$ & 5 \\
\hline 117 & H & 151.6 & $151 \cdot 6$ & $156 \cdot 3$ & $152 \cdot 5$ & 153.0 & 11.5 & 2.2 & 15304 & 11.4 & 2.1 & 5 \\
\hline 119 & 표 & 155.1 & 162.6 & $154 \cdot 5$ & 16103 & 158,4 & 1304 & $4 \cdot 2$ & 157.9 & 14.4 & 3.8 & 5 \\
\hline 120 & $\mathbf{R}$ & 161.2 & 155.4 & 156.6 & 148.1 & 155.3 & 16.0 & 5.4 & 153.8 & 15.8 & 5.5 & 5 \\
\hline 121 & $\mathbf{M}$ & 161.8 & 156.2 & 158.0 & 154.4 & 157.6 & 12.1 & 3.2 & 159.1 & 11.9 & 4.4 & 5 \\
\hline 123 & $\mathbb{R}$ & 148.7 & $150 \cdot 3$ & 147.6 & $142.5 X$ & $147 \cdot 3 x$ & 15.2 & 304 & $148 \cdot 4 X$ & 15.1 & 3.8 & 5 \\
\hline 125 & I & $156 \cdot 3$ & 158.5 & $155 \cdot 4$ & 154.6 & 156.3 & 10.3 & 109 & $156 \cdot 3$ & 1004 & 1.6 & 5 \\
\hline 127 & H & 156.3 & $156 \cdot 8$ & 150.4 & 157.9 & 1550 & 12.6 & 3.4 & 155.8 & 1109 & 3.1 & 5 \\
\hline 128 & H & $155 \cdot 1$ & 15406 & $152 \cdot 7$ & 153.8 & $154 \cdot 1$ & 1400 & 100 & 154.5 & 14.6 & $1 \cdot 3$ & 5 \\
\hline 129 & $\boldsymbol{R}$ & 152.79 & 156.3 & 149.0 & $15 S_{0} 5$ & 15404 & 11.4 & 405 & $150 \cdot 7$ & 10.5 & S. $2 x$ & 5 \\
\hline 130 & म & 151.4 & 162.7 & $15 s_{0} 5$ & $150 \cdot 35$ & 156.0 & 17.4 & 6.1 & 15504 & 17.0 & 5.4 & 5 \\
\hline 131 & $\mathbf{R}$ & 159.1 & $15 e_{0} 7$ & 150.6 & $15 \varepsilon_{\bullet} 7$ & 156,8 & 19.3 & 4.1 & 156.3 & $1 \varepsilon_{0} 4$ & 308 & 5 \\
\hline 133 & $\mathbf{A}$ & 156.4 & $154 \cdot 5$ & $144.9 X$ & 158.0 & 153.5 & 1207 & 509 & 15404 & 13.4 & $5 \cdot 5$ & 5 \\
\hline 134 & B & 148.5 & 156.2 & 153.5 & 158.8 & $154 \cdot 3$ & 11.8 & 4.4 & $154 \cdot 1$ & 11.4 & 3.8 & 5 \\
\hline 135 & I & 162.0 & 148.5 & 154.3 & 158.1 & 155.7 & 15.3 & 5.8 & 160,0 & 15.7 & $10.9 x$ & 5 \\
\hline 136 & B & 154.7 & $145,1 x$ & 151.7 & 148.7 & 150.0 & 12.2 & 4.1 & $14906 *$ & 11.8 & 3. 7 & 5 \\
\hline 137 & H & 152.7 & 154.7 & $161 \cdot 25$ & 156.6 & $156 \cdot 3$ & 16.2 & 3.6 & 157.6 & 16.9 & $4 \cdot 3$ & 5 \\
\hline 138 & B & $15 s \cdot 6$ & 156.1 & $1 \in 3,5$ & 159.2 & 159.6 & 16.1 & 300 & 158.9 & 15.8 & 3.0 & 5 \\
\hline 139 & $\mathbf{R}$ & 156.3 & $167 \cdot 6 x$ & 157.0 & 156.7 & 159.4 & 15.7 & 5.5 & 158.6 & 15.6 & 5.1 & 5 \\
\hline 140 & 표 & 154.5 & $156 \cdot 3$ & $153 \cdot 3$ & 162.8 & 156.7 & 13.9 & 402 & 155.5 & 12.8 & 4.6 & 5 \\
\hline 141 & H & $153 \cdot 3$ & 155.8 & $155 \cdot 2 \mathrm{~s}$ & $157 \cdot 0$ & 155.4 & $8 \cdot 4$ & 1.6 & 155.7 & e.1 & $1 \cdot 5$ & 5 \\
\hline 142 & $\mathbf{A}$ & 157.0 & $146 \cdot 2$ & $151 \cdot 3$ & 152.1 & 151.7 & 1109 & 404 & 151.4 & 12.6 & 309 & 5 \\
\hline 143 & H & 155.6 & $15 e \cdot 6$ & 155.2 & 156.6 & 156.5 & 15.4 & 1.5 & 157.0 & 1409 & 1.7 & 5 \\
\hline 145 & H & $153 \cdot 1$ & 153.0 & 154.5 & $157 \cdot 3$ & 154.5 & 11.7 & 2.0 & $154 \cdot 5$ & 11.7 & 1.7 & 5 \\
\hline 147 & E & $156 \cdot 8$ & $155 \cdot 2$ & 153.5 & 145.7 & 153.8 & 15.1 & 3.0 & 152.9 & 15,2 & $3 \cdot 3$ & 5 \\
\hline 149 & E & $159 \cdot 1$ & 156.6 & 160.6 & $169.8 X$ & 161.5 & 16.4 & 5.8 & $161.3 *$ & 17.3 & 5.0 & 5 \\
\hline 151 & ㅍ & $15 \epsilon .5$ & 156.8 & $154 \cdot 1$ & 156.8 & 156.0 & 9.9 & 1.3 & 156.1 & 10.5 & 1.1 & 5 \\
\hline 153 & H & $151 \cdot 3$ & 158.9 & $158 \cdot 2$ & 156.6 & $156 \cdot 3$ & 1502 & 304 & 156.3 & 15,2 & 3.4 & 4 \\
\hline 155 & H & 155.9 & 157.8 & 161.8 & 163.8 & 15909 & 18.3 & 3.6 & 159.5 & $1 \varepsilon_{0} 2$ & 3.2 & 5 \\
\hline 157 & $\cdot \mathrm{X}$ & 160.7 & 149.9 & 154.4 & 157.4 & 155.6 & 12.0 & 4.6 & 155.7 & 1209 & 4.0 & 5 \\
\hline 161 & $\cdot x$ & 156.9 & 158.6 & 156.8 & $15 \varepsilon_{0} 4$ & 157.7 & 15.4 & 1.0 & 157.1 & 14.2 & 1.5 & 5 \\
\hline 163 & H & 153.0 & 155.6 & 158.7 & 14904 & $154 \cdot 2$ & 16.2 & 3.9 & $154 \cdot 2$ & 16.2 & 3.9 & 4 \\
\hline 165 & $\mathbf{R}$ & 149.0 & 151.4 & 153.0 & 146.4 & 150.0 & 13.7 & 2.9 & $149.9 *$ & 13.8 & 2.5 & 5 \\
\hline 166 & B & & & & 167.4 & $167 \cdot 4 X$ & 14.3 & & $163 \cdot 4 x$ & 13.2 & 5.7 & 2 \\
\hline 167 & B & $1 \leqslant 5 \cdot 4$ & 160,0 & 158.7 & 156.4 & 157.6 & 11.9 & $2 \cdot 1$ & 159.4 & 1201 & $4 \cdot 3$ & 5 \\
\hline 169 & $I$ & 156.8 & 155.8 & 155.0 & $145 \cdot 6$ & $154 \cdot 3$ & 1504 & $3 \cdot 2$ & 155.8 & 15.6 & $4 \cdot 4$ & 5 \\
\hline 171 & E & $166 \cdot 6 x$ & $162 \cdot 2$ & $176.4 X$ & 161.7 & $166.8 x$ & 15.3 & 6.8 & $166.5 X$ & 16.3 & 5.9 & 5 \\
\hline 172 & 표 & 157.1 & 149.0 & $154 \cdot 4$ & 149.2 & 152.4 & 13.1 & 4.0 & $153 \circ 9$ & 13.7 & 4.7 & 5 \\
\hline 173 & H & 154.1 & 153.4 & 149.2 & 155.8 & $153_{0} 1$ & 10.4 & 2.8 & $153 \cdot 3$ & 10,5 & 2.5 & 5 \\
\hline 174 & H & 160.2 & $155 \cdot 2$ & $156 \cdot 2$ & 155.6 & $157 \cdot 8$ & $15 \cdot 4$ & 2.5 & $157 \cdot 8$ & 15.8 & 2.1 & 5 \\
\hline 175 & H & 156.7 & 158.7 & 155.9 & $157 \cdot 8$ & 157.3 & $13 \cdot 3$ & 1.2 & 156.8 & 1401 & 1.5 & 5 \\
\hline 176 & H & 162.6 & $157 \cdot 9$ & $149 \cdot 3$ & 143.7 & $153 \cdot 4$ & 14.9 & 8.5 & 15309 & 15.6 & $7.5 x$ & 5 \\
\hline
\end{tabular}




\begin{tabular}{|c|c|c|c|c|c|c|c|c|c|c|c|c|}
\hline \multicolumn{3}{|c|}{ LAB } & \multicolumn{3}{|c|}{ MEANS THIS MONTH } & \multicolumn{3}{|c|}{ THIS MENTH } & \multicolumn{4}{|c|}{ CUMULATIVE } \\
\hline CODE & $\mathbf{v}$ & $n x-1$ & W $\mathrm{K}-2$ & $n R-3$ & $\mathbf{W}=4$ & MEAN & SDR & SDWRS & MEAN & SDR & SDABS & WRS \\
\hline 177 & H & 159.0 & 158.5 & $161 \cdot 9$ & 158.9 & 159.6 & $14 \cdot 1$ & 1.6 & 158.5 & 14.3 & 2.8 & 5 \\
\hline 182 & $\mathbf{H}$ & 162.6 & 157.5 & 154.75 & 143.8 & $154 \cdot 8$ & 17.9 & 8,0 & $155 \cdot 4$ & 17.6 & $7 \cdot 1 x$ & 5 \\
\hline 184 & $\mathbf{B}$ & 157.2 & $155 \cdot 1$ & $150 \cdot ?$ & $155 \cdot 4$ & $154 \cdot 6$ & 13.6 & $2 \cdot 8$ & $154 \cdot 6$ & 13.6 & 2.8 & 4 \\
\hline 186 & H & $153 \cdot 2$ & $154 \cdot 5$ & 157.7 & $154 \cdot 6$ & 155.0 & 12.5 & 1.9 & $153 \cdot 3$ & 13.0 & 4.2 & 5 \\
\hline 188 & $I$ & 154.1 & $154 \cdot e$ & $156 \cdot 3$ & 155.9 & 155.3 & 12.7 & 1.0 & 155.7 & 12.4 & $1 \cdot 2$ & 5 \\
\hline 250 & $\bullet I$ & 177 . ex & $155 \cdot 2$ & 156.7 & 153.05 & 160.7 & 20.5 & 11.5 & $163 \cdot 3 x$ & 15.7 & $11.6 x$ & 5 \\
\hline 274 & H & 154.7 & $157 \cdot 8$ & 156.0 & $156 \cdot 4$ & 156.2 & 12.0 & $1 \cdot 3$ & 156.6 & 1108 & $1 \cdot 3$ & 5 \\
\hline 283 & H & $154 \cdot 2$ & $156 \cdot 3$ & 154.9 & $15 \varepsilon, 6$ & 156.0 & 10.7 & 1.9 & 156.0 & 10.6 & 1.7 & 5 \\
\hline 287 & $\mathbf{A}$ & $17 e_{0} 7 x$ & $167.0 x$ & $176.4 x$ & $176 \cdot 9 x$ & $174 \cdot 8 x$ & 17.0 & 5.3 & $171 \cdot 2 x$ & 15.6 & S. $1 x$ & 5 \\
\hline 350 & H & 157.6 & 152.8 & 153.1 & 145.5 & 152.3 & 15.0 & 5.0 & 153.2 & 15.5 & 4.8 & 5 \\
\hline 553 & $M$ & $161 \cdot 7$ & $157 \cdot 5$ & 159.7 & 160.9 & $160_{0} 0$ & 11.9 & 1.9 & $160_{0} 3$ & 12.3 & 1.7 & 5 \\
\hline 562 & $\mathbf{A}$ & $176,4 x$ & $166.7 x$ & $169.3 x$ & 166.6 & 165. $8 X$ & 16.5 & 4.6 & $16903 x$ & 16.4 & $4 \cdot 1$ & 5 \\
\hline 568 & $\mathbf{I}$ & $156 \cdot 7$ & 150.3 & 158.6 & $152 \cdot 7$ & 154.6 & 14.2 & $3 \cdot 7$ & 154.0 & 1403 & 3.5 & 5 \\
\hline 569 & $\boldsymbol{A}$ & 156.3 & 153.5 & 158.8 & 160.6 & $157 \cdot 3$ & 14.8 & $3 \cdot 1$ & 157.1 & 1402 & 2.7 & 5 \\
\hline 658 & H & 160.8 & 162.7 & 163.9 & 16601 & $163.4 X$ & 16.8 & $2 \cdot 2$ & 162.7 * & 15.8 & 2.4 & 5 \\
\hline 682 & $\mathbf{H}$ & $165_{\bullet} 0 x$ & $177 \cdot 2 x$ & $174 \cdot 7 x$ & $180,0 x$ & $174 \cdot 2 x$ & 14.8 & 6.5 & $174.2 X$ & 14.8 & 6.5 & \\
\hline 701 & H & $151 \cdot 5$ & 154.6 & $151 \cdot 3$ & & 152.5 & 17.7 & 1.8 & 151.7 & 17.0 & $2 \cdot 2$ & \\
\hline
\end{tabular}

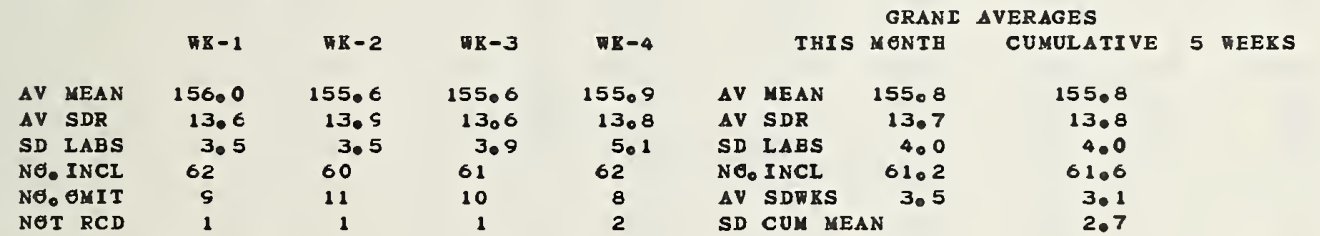


FIAT CRUSB STRENGTB (CONCORA), IB

\begin{tabular}{|c|c|c|c|c|c|c|c|c|c|c|c|}
\hline$I \Delta B$ & \multicolumn{4}{|c|}{ MEANS TBIS MENTE } & \multicolumn{3}{|c|}{ THIS MONTH } & \multicolumn{4}{|c|}{ CUNUL AT I VE } \\
\hline $\operatorname{CODE} V$ & M E - 1 & $w K-2$ & $M E-3$ & $m E-4$ & MEAN & SDR & SDWKS & MEAN & SDR & SDWRS & $\mathbf{m B S}$ \\
\hline 100 & 74.0 & $74 \cdot 1$ & 74.1 & $73 \cdot 2$ & 73. 8 & $4 \cdot 5$ & .4 & $73 \cdot 7$ & $4 \cdot 1$ & .5 & 8 \\
\hline 102 & & 72.5 & $72 \cdot 5$ & $72 \cdot 4$ & 72.5 & 2.5 & .1 & 72.6 & 2.9 & .5 & 7 \\
\hline 105 & 74.7 & $77 \cdot 8$ & $79,6 x$ & $81.9 x$ & 78.5 & 4.5 & 3.0 & 76.8 & 4.2 & 2,9 & 8 \\
\hline 106 & 72.5 & 72.6 & $72 \cdot 7$ & 73.8 & 72.9 & 3. 6 & .6 & $73 \cdot 2$ & 3.5 & $\bullet 8$ & 8 \\
\hline 110 & $72 \cdot 2$ & $73 \cdot 8$ & $72 \cdot 5$ & 73.6 & 73.0 & $3 \cdot 5$ & - 8 & $73 \cdot 6$ & 3.6 & $1 \cdot 1$ & 8 \\
\hline 113 & 71.2 & 72.0 & 71.6 & 71.6 & 71.6 & 2.5 & $\cdot 3$ & $71 \cdot 9$ & 2.8 & -4 & 8 \\
\hline 114 & 71.0 & $6 e .7$ & 71.7 & 70.5 & 70.5 & 3.1 & 1.3 & 70.7 & 3.2 & $1 \bullet 0$ & 8 \\
\hline 115 & 73.9 & $72 \cdot 5$ & 75.0 & 74.1 & 73.9 & $3 \cdot 3$ & 1.0 & 74.5 & 3.5 & $1 \cdot 2$ & 7 \\
\hline 119 & 70.8 & 67.5 & 69.9 & 69.3 & 69.4 & 3.5 & 1.4 & $69 \cdot 9$ & $4 \cdot 5$ & $1 \cdot 5$ & 8 \\
\hline 120 & 70.7 & 68.4 & 71.4 & 69.9 & 70.1 & 3.0 & $1 \cdot 3$ & 70.2 & $3 \cdot 3$ & 1.1 & 7 \\
\hline 125 & $79 \cdot 3 x$ & $75 \cdot 3$ & 76.5 & $77 a b$ & 77.2 & 4.8 & $1 \cdot 7$ & 78.8 * & $5.5 x$ & $2 \cdot 3$ & 8 \\
\hline 128 & 72.4 & 74.1 & 72.7 & 74.2 & 73.4 & 3,3 & .9 & 72.8 & 304 & 1.0 & 8 \\
\hline 136 & 76.1 & $74 \cdot 5$ & 73.7 & 70.3 & 73. 7 & $4 \cdot 3$ & 2.4 & 73.7 & 4.3 & 2.4 & 4 \\
\hline 138 & 77.1 & 77.25 & 73.5 & 74.8 & 75.7 & 4.5 & 1.8 & 75.2 & 4.5 & 1.7 & 7 \\
\hline 140 & $7 e .1$ & $80.2 x$ & 78.3 & 78.1 & 78.7 & 3.9 & 1.0 & $77.4 *$ & 4.4 & 1.7 & 8 \\
\hline 143 & $72 \cdot 2$ & 72.0 & $72 \cdot 1$ & $72 \cdot 3$ & 72.2 & 2.6 & .1 & 72.8 & 2.7 & .9 & 8 \\
\hline 151 & $63.4 x$ & 69.5 & 68.5 & & 67.1 & 3.5 & $3 \cdot 3$ & $67 \cdot 1$ * & 3.5 & 3.3 & 3 \\
\hline 161 & 71.5 & 71.6 & 72.8 & 74.0 & 72.5 & 4.8 & $1 \cdot 2$ & $73 \cdot 1$ & 4.9 & 209 & 8 \\
\hline 167 & 70.7 & 71.4 & 71.0 & 72.6 & 71.5 & 3.9 & $\cdot 8$ & 72.0 & 3.5 & 1.0 & 8 \\
\hline 177 & 72.2 & 69.6 & $72 \cdot 2$ & 71.4 & 71.4 & 3.7 & $1 \cdot 2$ & $71 \cdot 5$ & 308 & $1 \cdot 3$ & 8 \\
\hline 182 & 77.9 & 76.3 & 79.1 & $74 \cdot 2$ & 76.9 & 3.5 & $2 \cdot 1$ & $77 \cdot 6 *$ & 4.1 & 1.6 & 8 \\
\hline 168 & $73 \cdot 5$ & 72.8 & 71.8 & $7 c .9$ & 72.3 & 3.4 & 1.1 & 73.2 & 3.8 & $1 \cdot 5$ & 8 \\
\hline 250 & 71.8 & 72.4 & & 71.2 & 71.8 & 2.5 & .6 & 71.6 & 2.4 & .7 & 4 \\
\hline 269 & $65 \cdot 9$ & 73.9 & 72.2 & 71.8 & 72.0 & 304 & 1.6 & 72.0 & 3.7 & 1.2 & 8 \\
\hline 274 & 72.9 & 73.0 & $72 \cdot 6$ & $7 \geq .2$ & 72.9 & 3.0 & .3 & 73.0 & 300 & .2 & 8 \\
\hline 283 & 72,3 & 70.5 & 69.1 & 70.3 & 70.6 & 2.8 & 1.3 & 70.9 & 3.0 & $1 \cdot 3$ & 7 \\
\hline 284 & 71.6 & 70.5 & 71.9 & 72.8 & 71.7 & 3.2 & 1.0 & 71.3 & 3.6 & 1.7 & 8 \\
\hline 287 & 73.9 & 72.8 & 75.9 & 74.5 & $74 \cdot 3$ & 4.1 & $1 \cdot 3$ & $74 \cdot 5$ & 309 & $2 \cdot 2$ & 8 \\
\hline 289 & $55.8 x$ & $55 \cdot 6 x$ & $49 \cdot 8 x$ & $52 \cdot 3 x$ & $53.4 X$ & 2.9 & 2.9 & $60,5 x$ & 2.8 & e. ox & 8 \\
\hline 292 & $61.4 \mathrm{X}$ & 65.8 & 69.7 & 69.8 & 66.7 & 3.3 & 4.0 & $67.1 *$ & 3.5 & $4.5 x$ & 8 \\
\hline 350 & $88.4 \times S$ & $86 \cdot 3 x$ & $84.4 x$ & $82.2 x$ & $85.3 x$ & 5.0 & 2.6 & $84 \cdot 3 x$ & 4.6 & $4 \cdot 5 x$ & 8 \\
\hline 351 & 72.1 & 71.9 & $71 \cdot 3$ & 72.7 & 72.0 & 2.5 & .6 & 71.9 & 2.5 & .9 & 8 \\
\hline 353 & 74.7 & 71.0 & 74.4 & 74.6 & 73.7 & 4.1 & 1.8 & $72 \cdot 9$ & 3.6 & 1.6 & 8 \\
\hline 355 & 72.8 & 74.0 & 75.4 & 71.6 & 73.5 & $2 \cdot 8$ & 1.6 & 73.6 & 3.1 & 1.2 & 8 \\
\hline 357 & 74.0 & 76.1 & 77.5 & & 75.9 & $4 \cdot 2$ & 1.7 & 75.4 & 400 & 1.7 & 7 \\
\hline 361 & 72.7 & $68 \cdot 1$ & 70.9 & 70.2 & 70.5 & $3 \cdot 3$ & 1.9 & 70.7 & 3.0 & 1.4 & 8 \\
\hline 363 & 70.1 & 71.6 & 67.3 & 69.8 & 69.7 & 3.3 & 1.8 & 68.7 & 3.2 & 2.7 & 8 \\
\hline 365 & 71.6 & 72.1 & 68.4 & 71.6 & 70.9 & 3.1 & 1.7 & $70 \cdot 8$ & 3.1 & $1 \cdot 3$ & 8 \\
\hline 367 & $7 \geq .1$ & 74.6 & 70.8 & 71.9 & 72.6 & 4.1 & 1.6 & 73.7 & $4 \cdot 2$ & 2.0 & 8 \\
\hline 369 & 72.0 & 72.3 & 72.1 & 72.8 & $72 \cdot 3$ & $3 \cdot 2$ & 3 & 72.7 & 3.3 & .7 & 8 \\
\hline 377 & 76.7 & 76.3 & 73.4 & 76.7 & 75.8 & 3.9 & 1.6 & 75.5 & 4.0 & $1 \cdot 6$ & 7 \\
\hline 379 & 74.2 & & $75 \cdot 2$ & 72.0 & 74.0 & 3.8 & 1.7 & $73 \cdot 2$ & $4 \cdot 3$ & $1 \cdot 3$ & 7 \\
\hline 381 & & 71.9 & $71 \cdot 4$ & 70.6 & $71 \cdot 3$ & 3.8 & .7 & $71 \cdot 2$ & 3.7 & .7 & 6 \\
\hline 383 & 75.2 & $82.5 \times S$ & $82.1 \mathrm{X}$ & 75.1 & $78 \cdot 8$ & 4.7 & $4 \cdot 2$ & $75 \cdot 3$ & 4.0 & $4 \cdot 7 x$ & 8 \\
\hline 385 & $81.0 \mathrm{x}$ & 72.6 & $71.0 \mathrm{~s}$ & 74.0 & 74.7 & $5 \cdot 1$ & 4.4 & 74.1 & 4.8 & $3.5 x$ & 8 \\
\hline 387 & 71.3 & $75 \cdot 5$ & 74.4 & 73.3 & 73.6 & 4.0 & 1.8 & 73. 1 & 4.0 & 1.9 & 8 \\
\hline 391 & 67.4 & $61.9 x$ & 71.2 & $67 \cdot 3$ & 66.9 & 2.8 & $3 \cdot 8$ & $67 \cdot 2 *$ & 3.0 & $2 \cdot 5$ & 8 \\
\hline 393 & 70.8 & 69.9 & 71.0 & 70.1 & 70.5 & $3 \cdot 2$ & .5 & $71 \cdot 1$ & 309 & - 8 & 8 \\
\hline 395 & 73.0 & 74.0 & 72.9 & 74.2 & 73.5 & $3 \cdot 3$ & .7 & 73.1 & 305 & $1 \cdot 0$ & 8 \\
\hline 397 & 73.6 & 74.1 & 75.4 & 75.9 & 74.7 & 3.8 & $1 \cdot 1$ & 74.1 & 3.6 & $1 \cdot 2$ & B \\
\hline 399 & 73.8 & 73.8 & $74 \cdot 6$ & 73.9 & 74.0 & 4.1 & .4 & 73.8 & 3.9 & .9 & 8 \\
\hline 553 & 74.3 & 71.2 & 71.9 & 72.1 & 72.4 & 2.7 & $1 \cdot 3$ & 72.6 & 2.7 & $1 \cdot 1$ & 8 \\
\hline 555 & $7 \geq \cdot 2$ & 75.4 & $75 \cdot 5$ & 74.3 & 74.6 & 2.5 & $1 \cdot 1$ & 74.6 & 2.5 & 2.3 & 8 \\
\hline 562 & 72.8 & 71.3 & 70.8 & 72.8 & 71.9 & 3.1 & 1.0 & 71.3 & 3.7 & 1.4 & 8 \\
\hline 568 & 68.3 & 65.7 & 70.6 & 66.9 & 67.9 & 3.7 & 2.1 & 70.2 & 3.5 & 2.9 & 8 \\
\hline
\end{tabular}


FLAT CRUSH STRENGTH ( CONCORA), IB

\begin{tabular}{|c|c|c|c|c|c|c|c|c|c|c|c|}
\hline \multirow{2}{*}{$\begin{array}{l}I A B \\
C O D E\end{array}$} & \multicolumn{4}{|c|}{ MEANS IBIS MENTH } & \multicolumn{3}{|c|}{ THIS MONTH } & \multicolumn{4}{|c|}{ CUMULATIVE } \\
\hline & $m B-1$ & $\nabla x-2$ & $\bar{n} \boldsymbol{I}-3$ & m $x-4$ & MEAN & SDR & SDTES & MEAN & SDR & SDMES & WBS \\
\hline 572 & 78.5 & $75 \cdot 2$ & 74.7 & 70.6 & $74 \cdot 8$ & 4.0 & $3 \cdot 2$ & 75.3 & $3 \cdot 8$ & 2.5 & 8 \\
\hline 578 & 76.7 & 69.5 & $40.9 x$ & 73.8 & $65 \cdot 2 x$ & $4 \cdot 4$ & 16.5 & $67.4 \div$ & 4.7 & $12.4 x$ & 8 \\
\hline 609 & $76 \cdot 2$ & $74 \cdot 5$ & 72.5 & 70.9 & $73 \cdot 6$ & 3.7 & 2.3 & $71 \cdot 5$ & $3 \cdot 8$ & 2.9 & 8 \\
\hline 614 & 69.4 & 69.6 & 66.5 & & 68,7 & 3.4 & 1.5 & 68.7 & $3 \cdot 4$ & 1.5 & 3 \\
\hline 617 & $81.0 \times 5$ & $85 \cdot 1 \times 5$ & $82 \cdot 5 \times s$ & $80 \cdot 0 x$ & $82.2 x$ & $6 \cdot 3$ & 2.2 & $81.9 x$ & $6.7 x$ & $2 \cdot 8$ & 8 \\
\hline
\end{tabular}

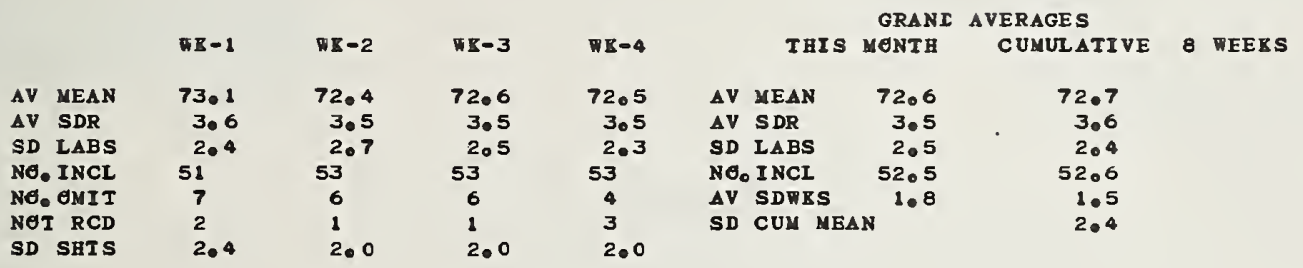


NBS.114A (REV. 9-78)

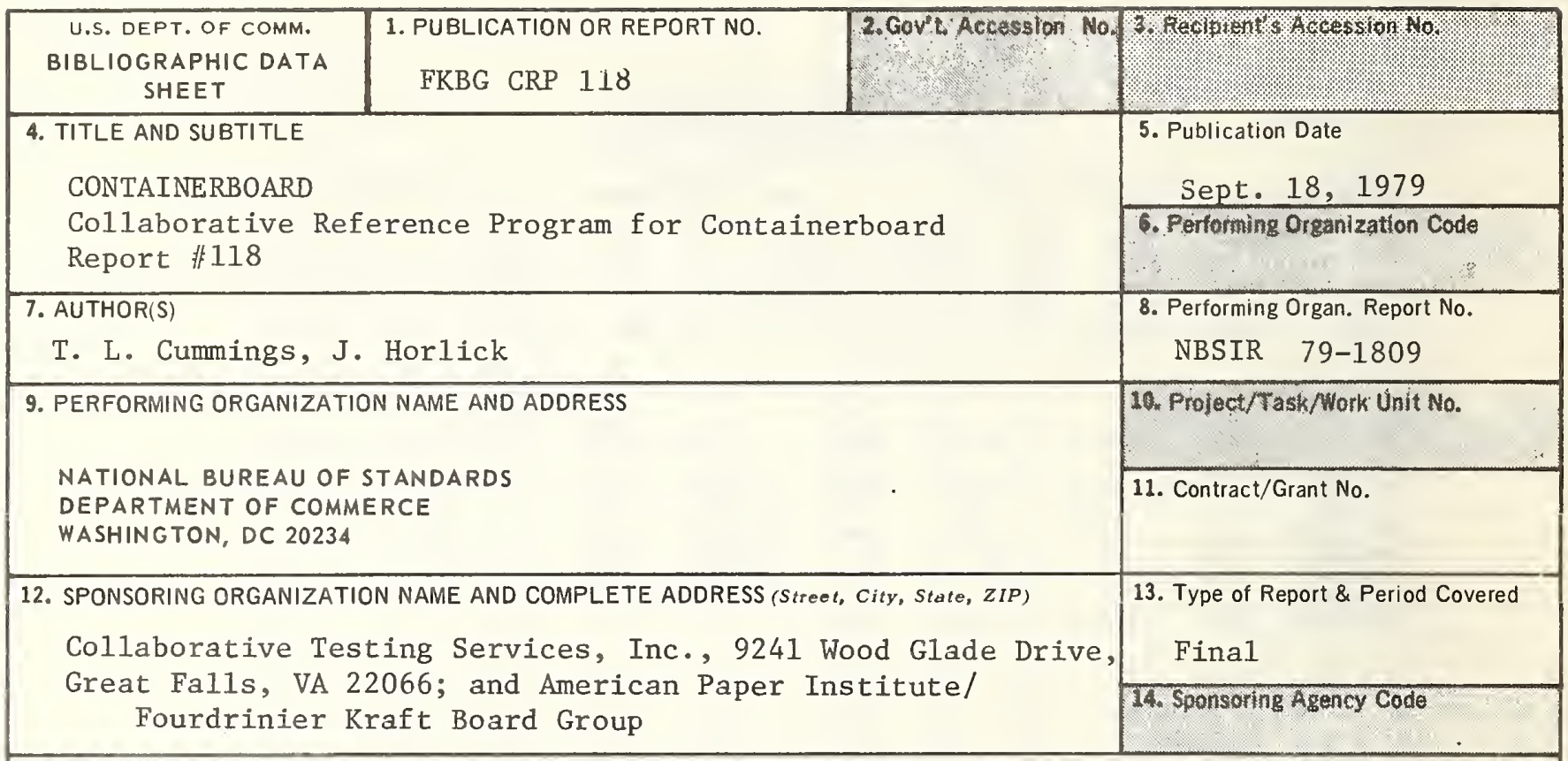

15. SUPPLEMENTARY NOTES

Document describes a computer program; SF-185, FIPS Software Summary, is attached.

16. ABSTRACT (A 200-word or less factual summary of most significant information. If document includes a significant bibliography or literature survey, mention it here.)

Collaborative Reference Programs provide participating laboratories with the means for checking periodically the level and uniformity of their testing in comparison with that of other participating laboratories. An important by-product of the programs is the provision of realistic pictures of the state of the testing art. This is one of the periodic reports showing averages for each participant, within and between laboratory variability, and other information for participants and standards committees.

17. KEY WORDS (six to twelve entries; alphabetical order; capitalize only the first letter of the first key word unless a proper name; separated by semicolons)

Collaborative reference program; Containerboard; Laboratory evaluation;

Precision; Reference samples; Testing calibration
18. AVAILABILITY

$\square$ Unlimited

X For Official Distribution. Do Not Release to NTIS

Order From Sup. of Doc., U.S. Government Printing Office, Washington, DC 20402, SD Stock No. SN003-003-

$\square$ Order From National Technical Information Service (NTIS), Springfield, VA. 22161

\begin{tabular}{|l|c|}
\hline $\begin{array}{l}\text { 19. SECURITY CLASS } \\
\text { (THIS REPORT) } \\
\text { UNCLASSIFIED }\end{array}$ & $\begin{array}{c}\text { 21. NO. OF } \\
\text { PRINTED FAGES }\end{array}$ \\
\hline $\begin{array}{l}\text { 20. SECURITY CLASS } \\
\text { (THIS PAGE) }\end{array}$ & 22. Price \\
UNCLASSIFIED & \\
\hline
\end{tabular}




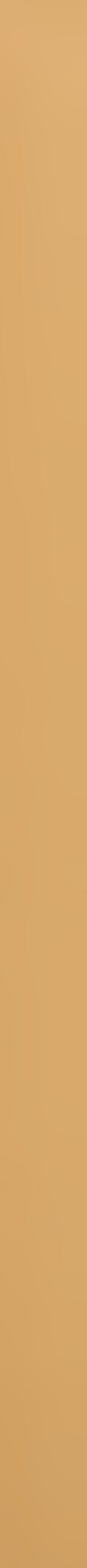


